\title{
Estrategias de evaluación en la enseñanza de los algoritmos de factorización en noveno grado de Educación Secundaria
}

\author{
Nicolaza del Carmen Flores Medina ${ }^{1}$ \\ Mariana Lisseth Pastrana Jarquín ${ }^{2}$ \\ William Oswaldo Flores López ${ }^{3}$
}

\section{Resumen}

En este artículo se han descrito las estrategias de evaluación en la enseñanza de los algoritmos de factorización en noveno grado de Educación Secundaria. Se trató de un estudio cualitativo sustentado en un diseño fenomenológico donde se suministró una entrevista a siete profesores de matemáticas de Educación Secundaria con el propósito de detallar las experiencias individuales subjetivas de los participantes sobre la evaluación. Los principales resultados afirman que el profesorado evalúa la enseñanza de la factorización con: estrategias metodológicas, recursos didácticos, tecnológicos, criterios e instrumentos de evaluación y registro de evaluación diagnóstica. Concluyendo que, la evaluación de aprendizajes es útil, flexible y válida para la toma de las decisiones en la vinculación de los cognitivo y afectivo, así como, para proponer situaciones didácticas que mejoren el aprendizaje de las matemáticas del estudiantado.

Palabras clave: estrategia de evaluación; algoritmo de factorización; enseñanzaaprendizaje.

\section{Abstract}

In this article have been described the assessment strategies of the factorization algorithms in ninth grade of Secondary Education. It was about a qualitative study supported by a phenomenological design, where an interview was given to seven Secondary Mathematics teachers with the purpose of specifying the individual subjective experiences of the participants of the assessment. The main results state that the teacher evaluates the teaching of factorization with methodological strategies, didactic resources, technological, criteria and instruments of evaluation and registry of diagnostic evaluation. In conclusion, the evaluation of learning is useful, flexible

1 Licenciada en Educación Media con Mención en Matemática. Profesora de Matemáticas, MINED-Nueva Guinea. Correo: ncarmenfm17@gmail.com

2 Licenciada en Educación Media con Mención en Matemática. Profesora de Matemáticas, MINED-Nueva Guinea. Correo: pastranamariana@gmail.com

3 Doctor en Educación, Profesor Investigador de la Universidad de las Regiones Autónomas de la Costa Caribe Nicaragüense. Correo: william.flores@uraccan.edu.ni 
and valid for making decisions in the linking of cognition and affectivity, as well as, to propose teaching situations that improve the learning of students' mathematics.

Keywords: assessment strategy, factorization algorithm, teaching learning

\section{Introducción}

Este trabajo se centra en describir las estrategias de evaluación en la enseñanza de los algoritmos de factorización en noveno grado de Educación Secundaria. Para ello, se aplicó una entrevista al profesorado, que permitió categorizar las estrategias de evaluación en términos de: metodologías, criterios, instrumentos, registro, medidas, utilidad y vinculación con la evaluación de los aprendizajes. El estudio de las estrategias de evaluación de los aprendizajes en la factorización de expresiones algebraicas servirá de ayuda para proponer situaciones didácticas que mejoren el aprendizaje de las matemáticas del estudiantado.

En la literatura ya existen estudios que tratan de clasificar estrategias de evaluación e identificar su importancia. Por ejemplo, Flores y López (2016) afirman que el profesorado de matemáticas promueve estrategias de evaluación estimuladoras y participativas que conllevan a prácticas interactivas con grupos interactivos en aula de clases, lo que contribuye a un aprendizaje significativo en matemáticas.

En la comprensión que la evaluación de los aprendizajes deber ser un proceso sistemático y permanente que comprende la búsqueda y obtención de información de diversas fuentes acerca de la calidad, desempeño, avance, rendimiento o logro del estudiantado y de calidad de los procesos empleados por el profesorado, la determinación de su importancia y pertinencia de conformidad con los objetivos de formación que se espera alcanzar (URACCAN, 2007). La evaluación de los aprendizajes se caracteriza por estar inmersa en el desarrollo habitual del proceso de enseñanza-aprendizaje; por abarcar todo el proceso formativo, inicial, de proceso y final; ser formativa; y por incluir demandas cognitivas variadas y progresivas (Zabalza, 2001).

\section{Revisión de la literatura}

La factorización es una técnica que consiste en la descomposición de una expresión matemática que puede ser un número, una suma o resta, una matriz u polinomio en forma de producto. Existen distintos métodos de factorización, dependiendo de los objetos matemáticos estudiados, el objetivo es simplificar una expresión o reescribirla en "bloques fundamentales", en particular factorizar un polinomio consiste en expresarlo como un producto de otros polinomios, cada polinomio en el producto es un factor del polinomio original. 
Según Jarquín (2009), la enseñanza de la factorización en noveno grado consiste en aplicar procedimientos de factorización, identificando las características de cada caso. Tomando en cuenta el respeto a la diversidad y a la dignidad humana al relacionarse con las personas en un ambiente pluralista a fin de contribuir a una cultura de paz. Lo que se traduce en indicadores de logros como: establece la relación entre factorización y productos notables; diferencia y realiza un factor común monomio y factor común polinomio; expresa la diferencia de cuadrados como el producto de dos factores; y factorización sumas y diferencias de cubos, trinomios cuadrados perfectos, trinomios de la forma $a x^{2}+b x+c$ y polinomios de la forma $x^{2} \pm 3 x^{2} y+3 x y^{2} \pm y^{3}$ (Jarquín, 2009, pp. 146-151).

Los contenidos que se imparten en el noveno grado sobre factorización son: factor común y factor común polinomio; diferencia de cuadrados perfectos; suma y diferencia de cubos; trinomio cuadrado perfecto; trinomio de la forma $a x^{2}+b x+c$; y polinomio de la forma $x^{2} \pm 3 x^{2} y+3 x y^{2} \pm y^{3}$. Para Jarquín (2009) este indicador se valora a través de procedimientos de evaluación como: revisar el dominio del estudiantado sobre productos notables e identificar los que necesitan reforzamiento; verificar que el estudiantado establece una relación coherente, entre los tipos de factorización y su solución de acuerdo a sus propias características; observar y estimular la participación activa del estudiantado en cuanto al reconocimiento de la diferencia de cuadrados como el producto de dos factores; valorar conocimientos, dominio y motivación del estudiantado respecto a los tipos de factorización estudiado y las estrategias utilizadas para resolverlos; valorar el sentido de organización, respeto, colaboración, creatividad, originalidad, cientificidad y sentido de responsabilidad en el desarrollo y presentación de los trabajos (Jarquín, 2009, pp. 141-148).

\section{Materiales y métodos}

El presente estudio se enmarca en el enfoque cualitativo porque, "utiliza la recolección de datos sin medición numérica para descubrir o afinar preguntas de investigación en el proceso de interpretación" (Hernández, Fernández, \& Baptista, 2010, p. 7). En Bisquerra (2009) se afirma que la investigación cualitativa es una actividad sistemática, orientada a la comprensión en profundidad de fenómenos educativos y sociales, a la transformación de prácticas y escenarios socioeducativos, a la toma de decisiones y también hacia el descubrimiento y desarrollo de un cuerpo organizado de conocimiento.

A partir de las aportaciones de estos expertos, nuestra investigación se centrará en describir las estrategias de evaluación en la enseñanza de los algoritmos de factorización en noveno grado de Educación Secundaria. En Hernández, Fernández y Baptista (2010), se explica que en el enfoque cualitativo, el diseño o tipo de estudio "es el abordaje general que utilizará en el proceso de investigación" (p. 492). En este sentido, esta investigación tendrá un diseño o tipo de estudio fenomenológico, porque describe las experiencias individuales subjetivas de los participantes. 
Los participantes en esta investigación son siete profesores de matemáticas de Educación Secundaria que tienen entre 5 a 10 años de experiencia impartiendo matemática, principalmente el algoritmo de factorización algebraica. A estos profesores se les suministró una entrevista para que aportaran sobre las estrategias de enseñanza de la descomposición factorial algebraica, así como los procedimientos de evaluación que implementan para verificar el aprendizaje de este contenido matemático. El procesamiento de análisis se efectuó de manera cualitativa usando una matriz que permitió la categorización de la metodología de enseñanza, así como las estrategias de evaluación que el profesorado implementa.

\section{Resultados}

La factorización juega un papel muy importante en la formación del estudiantado, porque está presente en muchos contenidos básicos de la educación secundaria, así como en contenidos matemáticos de la educación superior. Por ello, el profesorado de matemática que trabaja en educación secundaria debe tener bastante experiencia para explicar correctamente el sentido lógico de las estructuras algebraicas, es importante que el estudiantado domine correctamente las operaciones básicas aritméticas y algebraicas. La estrategia metodológica en la enseñanza del algoritmo de factorización que implementa el profesorado se caracteriza por aprendo, practico y aplico, sustentadas en estrategias de enseñanza y la utilización de recursos didácticos y tecnológicos.

Tabla 1: Estrategias metodológicas en la enseñanza de los algoritmos de factorización

\begin{tabular}{|c|c|c|}
\hline Estrategias de Enseñanza & & $\begin{array}{c}\text { Recursos Didácticos y } \\
\text { Tecnológicos }\end{array}$ \\
\hline $\begin{array}{l}\text { - } \text { Resolución de problemas expositiva- } \\
\text { mente. } \\
\text { - } \text { Relacionar con el producto notable. } \\
\text { - Lluvias de ideas. } \\
\text { - Enseñanza por métodos geométricos. } \\
\text { - Estudiantes monitores. } \\
\text { - Trabajos grupales. } \\
\text { - Clases expositivas. } \\
\text { - Descomponer factores en números. } \\
\text { - Mapas conceptuales. } \\
\text { - Debate. } \\
\text { - Experiencias previas. }\end{array}$ & $\begin{array}{c}\text { Estrategias } \\
\text { metodológicas en } \\
\text { la factorización }\end{array}$ & $\begin{array}{l}\text { - Videos. } \\
\text { - Uso de textos. } \\
\text { - Elaboración de fichas. } \\
\text { - Uso de la Pizarra. } \\
\text { - Las teselas con trozos de colores. } \\
\text { - Paginas con ejercicios } \\
\text { - Computadoras } \\
\text { - } \text { Rompecabezas } \\
\text { - Hojas con cuadrículas } \\
\text { - Celulares Tablet } \\
\text { - } \text { Aplicaciones Android } \\
\text { - Murales } \\
\text { - Papelones }\end{array}$ \\
\hline
\end{tabular}

Según el profesorado entrevistado las estrategias de enseñanza más utilizadas en la factorización es la resolución de problemas prácticos expositivamente, igualmente los métodos geométricos porque permiten explicar el sentido lógico de las estructuras algebraicas, así como la memorización de dichas estructuras. También, el profesorado 
enseña la factorización relacionándolos con los productos notables y mencionando las formas posibles para identificar cada caso de descomposición factorial. Algo importante a mencionar, es que el profesorado utiliza mapas conceptuales para explicar y demostrar las soluciones de los casos de descomposición algebraica.

El profesorado promueve la participación del estudiantado en la enseñanza de los algoritmos de factorización con estrategias como el trabajo en equipo, estudiantes monitores, lluvias de ideas, clases expositivas, debate, actividades en la pizarra y experiencias previas; contribuyendo a la construcción colectiva del conocimiento del estudiantado. Destacamos que el profesorado de matemática menciona que enseñar el algoritmo de factorización es más fácil y comprensible para el estudiantado a través de recursos didácticos y tecnológicos como: uso de texto, elaboración de fichas, uso de pizarra, las teselas con trozo de colores, páginas con ejercicios, hojas de cuadrículas, murales, rompecabezas, computadoras, celulares, Tablet, videos y aplicaciones Android.

Por otra parte, el profesorado comentó que los criterios de evaluación de los aprendizajes en esta temática deben ser definidos o redactados en función de: indicadores de logros; competencias, resultados de aprendizaje y resolución de sistemas de prácticas compartidas.

Tabla 2: Criterios e instrumentos de evaluación de los aprendizajes

\begin{tabular}{|c|c|c|}
\hline Criterios de evaluación & & Instrumento de evaluación \\
\hline $\begin{array}{l}\text { - En función del indicador de } \\
\text { logro a evaluar. } \\
\text { - En función de las competencias } \\
\text { a evaluar. } \\
\text { - En función de los resultados de } \\
\text { aprendizajes del estudiantado. } \\
\text { - En función de sistemas de prác- } \\
\text { ticas compartidas que realiza el } \\
\text { estudiantado. }\end{array}$ & $\begin{array}{l}\text { Criterios de } \\
\text { evaluación de los } \\
\text { aprendizajes }\end{array}$ & $\begin{array}{l}\text { - Rúbricas. } \\
\text { - Prueba escrita. } \\
\text { - Exposiciones. } \\
\text { - Conferencias. } \\
\text { - Tareas. } \\
\text { - Lista de cotejo. } \\
\text { - Trabajos guiados. } \\
\text { - Ejercicios grupales. } \\
\text { - Clases interactivas con } \\
\text { aplicaciones móviles. }\end{array}$ \\
\hline
\end{tabular}

Esta apreciación de evaluación de los aprendizajes del estudiantado es de forma individual o colectiva con la mediación del profesorado con el fin de obtener resultados más óptimos en el desarrollo de las competencias, habilidades, conocimientos, actitudes y valores. Por tanto, el profesorado construye instrumento y estrategias de evaluación de los aprendizajes como: rúbricas, pruebas escritas, exposición, conferencias, tareas, lista de cotejo, trabajos guiados, ejercicios grupales, y clases interactivas con aplicaciones móviles. Dicho proceso, parte de una planificación de la evaluación de los aprendizajes que exige la estructuración de aspectos didácticos mediante actividades prevista para desarrollar en un tiempo determinado y en un espacio definido y con el fin de conseguir los objetivos de aprendizajes previsto. 


\section{EDUCACIÓN}

Además, la relación de objetivos-actividades-contenidos es relevante en la planeación de la evaluación de los aprendizajes, porque configura los demás elementos como modos de interacción, mediaciones necesarias y formas de evaluación, a la vez, implica dar respuestas a cuestiones como: ¿Qué evaluaré? ¿Para qué evaluaré? ¿Cómo evaluaré? ¿Con qué instrumentos evaluaré? ¿Cuándo evaluaré? Según el profesorado, estas preguntas se formulan a lo largo de la planificación y evaluación de los aprendizajes correspondientes a sus momentos y actividades. Así mismo, el profesorado menciona que los criterios por considerar tienen que estar directamente relacionados con los objetivos propuestos, y, por otro lado, por los aspectos considerados en el diseño didáctico, los cuales deben ser consecuentes con lo estipulado en la fase de planeación.

En relación con los registros de evaluación diagnóstica para el profesorado es de carácter cualitativo registrándose en listas de cotejos, cuadernos de registros, cuaderno del estudiantado, en la planificación de los aprendizajes. El profesorado señala los resultados de la evaluación diagnóstica que se tipifica en las medidas que son utilizadas, por ejemplo: en el refuerzo a los contenidos con problemáticas de aprendizaje; la planificación de los aprendizajes con estrategias metodológicas que contribuyan a la construcción del concepto de factorización; en la retroalimentación del contenido; y en la readecuación del contenido con fines de una mejor apropiación del conocimiento. Finalmente, Ayuda al estudiantado a saber cómo mejorar, una buena retroalimentación (feedbacks) no solamente indica al estudiante en qué se equivocó, sino también en qué estuvo bien y, sobre todo, cómo puede mejorar aquello en que presentó debilidades.

Tabla 3: Registro de evaluación y medidas para su implementación

\begin{tabular}{|c|c|c|}
\hline Registro de la evaluación & & Medidas \\
\hline $\begin{array}{l}\text { - Cualitativamente a través de una lista } \\
\text { de cotejo. } \\
\text { - Cualitativamente para medir las difi- } \\
\text { cultades sobre las temáticas. } \\
\text { - Se registra en el cuaderno del estu- } \\
\text { diantado. } \\
\text { - Se registra en la planificación de los } \\
\text { aprendizajes. } \\
\text { - Cuaderno de registro de aprendizaje. }\end{array}$ & $\begin{array}{l}\text { Registro de } \\
\text { la evaluación } \\
\text { diagnostica }\end{array}$ & $\begin{array}{l}\text { - } \text { Refuerzos a los contenidos con } \\
\text { problemática de aprendizajes. } \\
\text { - } \text { Planificación de los aprendizajes } \\
\text { con estrategias metodológicas. } \\
\text { - Grupos monitores y reforzamiento. } \\
\text { - } \text { Retroalimentación del contenido. } \\
\text { - }\end{array}$ \\
\hline
\end{tabular}

Según el profesorado la utilidad de la evaluación de los aprendizajes se caracteriza por ser accesible, guiada, flexiva, construcción colectiva, y controlada por su avance progresivo. Por ello, los resultados de la evaluación del aprendizaje son elementos importantes, que conllevan a tomar decisiones como: adecuación de los contenidos, sistematización de las dificultades sobre la factorización, a una mejor apropiación de 
los aprendizajes, verificar el alcance de la apropiación de la factorización y ayuda a fortalecer las debilidades y dificultades de aprendizaje.

Tabla 4: Utilidad de la evaluación de los aprendizajes

\begin{tabular}{|c|c|c|}
\hline Flexible, útil y válida & & Toma de decisiones \\
\hline $\begin{array}{l}\text { - Accesible para la valoración de los } \\
\text { aprendizajes. } \\
\text { - Guiada a través de una programa- } \\
\text { ción de contenidos. } \\
\text { - Flexible porque valora las dificulta- } \\
\text { des de aprendizajes. } \\
\text { - Evaluación en proceso construyen- } \\
\text { do colectivamente. } \\
\text { - Control exhaustivo sobre un avance } \\
\text { progresivo. }\end{array}$ & $\begin{array}{c}\text { Utilidad de la } \\
\text { evaluación de los } \\
\text { aprendizajes }\end{array}$ & $\begin{array}{l}\text { - Ayuda a la adecuación de } \\
\text { los contenidos. } \\
\text { - Sistematiza las dificultades } \\
\text { de aprendizajes. } \\
\text { - Integra la participación del } \\
\text { estudiantado. } \\
\text { - A la apropiación del conte- } \\
\text { nido para un mejor apren- } \\
\text { dizaje. } \\
\text { - Verificar alcance de apro- } \\
\text { piación del conocimiento. } \\
\text { - Ayuda a fortalecer las de- } \\
\text { bilidades y dificultades del } \\
\text { estudiantado en relación } \\
\text { con los contenidos. }\end{array}$ \\
\hline
\end{tabular}

Para Flores (2017) los resultados de la evaluación deben llevarnos a aplicar medidas pertinentes y oportunas para mejorar el proceso de aprendizaje. Esto implica volver sobre lo actuado para atender aquellos aspectos que requieren reeducaciones, profundización, refuerzo o recuperación. Además, tomar decisiones es el objetivo mismo de la evaluación. Siempre hay decisiones que se desprenden de un proceso evaluador, buscando una mejora del programa, según la información analizada (añadir o quitar contenidos, cambiar la consecuencia de actividades, introducir actividades nuevas, proporcionar guiones que oriente el trabajo del estudiantado, reducir las pautas de orientación para favorecer la autonomía) y como parte de un procedimiento acreditativo o selectivo (dar paso a la siguiente fase y repetir...).

La evaluación es vinculante a los factores afectivos y cognitivos, porque según el profesorado, se debe despertar afectividad del tipo interés y motivación en el estudiantado para poder desarrollar la clase, potencializar el pensamiento crítico y razonamiento lógico. Además, lo afectivo se vincula con lo cognitivo en términos de situaciones didácticas agradables; prácticas matemáticas que den confianza; y problemas relacionados con la vida real y del contexto del estudiantado. 
Tabla 5: Vinculación de la evaluación de los aprendizajes

\begin{tabular}{|c|c|c|}
\hline Afectivo & & Cognitivo \\
\hline $\begin{array}{l}\text { - Estrategias de interés por el estudiantado. } \\
\text { - Estrategias de motivación por el estudiantado. } \\
\text { - } \text { Situaciones didácticas que le agraden al estu- } \\
\text { diantado. } \\
\text { - Con prácticas matemáticas que le den con- } \\
\text { fianza al estudiantado. } \\
\text { - Con un aprendizaje afectivo relacionado con } \\
\text { problemas de la vida real y del contexto }\end{array}$ & $\begin{array}{l}\text { Vinculación de } \\
\text { la evaluación de } \\
\text { los aprendizajes }\end{array}$ & $\begin{array}{l}\text { - Instructivo con lo educativo. } \\
\text { - } \text { Cognitivo con las TIC } \\
\text { - Integro con experiencia y } \\
\text { sistemas de prácticas. } \\
\text { - Integrado por estrategias } \\
\text { metodológicas }\end{array}$ \\
\hline
\end{tabular}

También, el profesorado incorpora aspectos instructivos y educativos, así como la integración de las TIC; las experiencias matemáticas; sistema de prácticas compartidas; y estrategias metodológicas. En síntesis, el profesorado durante el desarrollo de una actividad, plantea situaciones de choques cognitivos entre los estudiantes para tomar en cuenta los aspectos cognoscitivos, procedimentales y actitudinales.

\section{Conclusiones}

En esta investigación se han identificado las estrategias de evaluación de los aprendizajes en la enseñanza de los algoritmos de factorización. El análisis permite decir que las metodologías de enseñanza de la factorización en noveno grado se caracterizan por metodología de aprendo, practico y aplico sustentado en la resolución de problemas prácticos expositivamente; enseñanza por métodos geométricos; memorización de estructuras algebraicas; relaciones con los productos notables; mapas conceptuales. Además, se promueve la participación y estimulación a través de estudiantes monitores, lluvias de ideas, clases expositivas; debate, actividades en la pizarra; y experiencias previas que permiten la construcción colectiva de los conceptos, propiedades y procedimientos de la descomposición factorial algebraica. Se destaca que, el profesorado incorpora recursos didácticos y tecnológicos como uso de texto, elaboración de fichas, uso de pizarra, las teselas con trozo de colores, páginas con ejercicios, hojas de cuadrículas, murales, rompecabezas, computadoras, celulares, Tablet, videos y aplicaciones Android.

El profesorado utiliza criterios de evaluación en función de indicadores de logros, competencias, resultados de aprendizajes y resolución de sistemas de prácticas compartidas, concordando con los resultados de Amador, Reyes y Flores (2015) donde se dice que el profesorado de matemáticas planifica la evaluación de los aprendizajes a partir de competencias, ejes transversales, indicadores de logros, contenidos, actividades de aprendizajes y procedimientos de evaluación. Estos resultados se relacionan con la afirmación de Villa et al. (2013), el profesorado debe centrarse en lograr unos resultados de aprendizaje del estudiantado, con el fin de que éste llegue a dominar el desempeño de una o varias competencias. También, el profesorado utiliza instrumentos 
de evaluación y estrategias de evaluación como: rúbricas, pruebas escritas, exposición, conferencias, tareas, lista de cotejo, trabajos guiados, ejercicios grupales, y clases interactivas con aplicaciones móviles, armonizándose con los estudios de Flores y López (2016) que afirman que el profesorado de matemáticas incorpora instrumentos de evaluación con recursos didácticos y tecnológicos en el aprendizaje de las matemáticas.

En relación con los registros de evaluación diagnóstica el profesorado realiza de una forma cualitativa a través de lista de cotejos, cuadernos de registro, cuadernos del estudiantado y en la planificación de los aprendizajes. Igualmente, el profesorado utiliza los resultados de evaluación diagnóstica para la retroalimentación del contenido, la planificación de estrategias que favorezcan el aprendizaje y sobre todo la readecuación del contenido de factorización. Por otra parte, la utilidad de la evaluación de los aprendizajes se caracteriza por ser accesible, guiada, flexible, colectiva y controlada por su avance progresivo. Por ello, los resultados de la evaluación del aprendizaje son elementos importantes, que conllevan a tomar decisiones como: adecuación de los contenidos, sistematización de las dificultades sobre la factorización, a una mejor apropiación de los aprendizajes, verificar el alcance de la apropiación de la factorización y ayuda a fortalecer las debilidades y dificultades de aprendizaje.

La evaluación es vinculante en lo cognitivo y afectivo, representada en situaciones didácticas agradables; prácticas matemáticas que den confianza y problemas relacionados con la vida real y del contexto del estudiantado que permiten despertar la afectividad del tipo de interés, agrado, utilidad, confianza y motivación. Sabiendo que la resolución de cualquier problema matemático lleva asociada una situación afectiva para el sujeto implicado, quien pone en juego no solamente prácticas operativas y discursivas para dar una respuesta al problema, sino también moviliza creencias, actitudes, emociones y valores que condicionan en mayor o menor grado y diferente sentido la respuesta cognitiva requerida (Godino, 2013). Además, que el dominio afectivo hacia las matemáticas juega un papel importante en la evaluación de los aprendizajes. Ya que, un objetivo en la enseñanza y aprendizaje de las matemáticas, es que los estudiantes desarrollen actitudes, creencias y emociones que aumenten sus probabilidades de utilizar con éxito las matemáticas que saben y deben aprender más matemáticas (Flores \& Auzmendi, 2016).

Concluir, que la estrategias de evaluación tienen que ser asumidas, desde un contexto de poblaciones diversas, y se hace necesario considerar entre otros, los siguientes aspectos para mejorar el proceso evaluativos en el aula (Flores \& Auzmendi, 2015): (i) La importancia de la evaluación radica en el acercamiento del individuo a las normas y pautas culturales que permiten salvaguardar la identidad del pueblo; (ii) Una de las características de la evaluación para poblaciones indígenas, afrodescendientes y mestiza es que da la relación estrecha de padre-hija, madre-hija y otros parientes en el momento mismo en el que el hijo o la hija realiza determinada actividad, se evalúa diariamente; (iii) La evaluación para este tipo de poblaciones también se caracteriza 


\section{EDUCACIÓN}

por su carácter cualitativo y es evaluación integral porque se consideran todos los ámbitos de la vida del niño o niña: afectivo, cognoscitivo y psicomotor. (iv) El aspecto valorativo en la evaluación para este contexto diverso es una responsabilidad colectiva, pues participan todos los miembros de la comunidad, tanto mayores como grupo de igual edad; y (v) Es así como los niños se enfrentan a dos paradigmas: la escuela sanciona y la educación en contexto de diversidad pretende hacer personas mejores, capaces de dar solución a problemas y desenvolverse en su medio.

\section{Lista de referencias}

Amador, F., Reyes, M., \& Flores, W., O. (2015). Metodologías en la enseñanza del cálculo de probabilidades en undécimo grado de educación secundaria. Ciencia e Interculturalidad, 17(2), 15-27.

Bisquerra, R. (2009). Metodología de la Investigación Educativa. Madrid: La Muralla.

Flores, W., O. (2017). Sistema de evaluación de los aprendizajes en el modelo de Universidad Comunitaria Intercultural. Managua: URACCAN.

Flores, W., O., \& Auzmendi, E. (2015). Integración de las tecnologías de la información y comunicación en la formación de profesores de matemática en y para la diversidad. En L. Bengochea, C. Varela, \& A. Miñan (Ed.), Congreso Internacional sobre Formación Virtual Inclusiva y de Calidad para el Siglo XXI (281-288). Granada: Universidad de Granada.

Flores, W., O., \& Auzmendi, E. (2016). Los problemas de comprensión del álgebra en estudiantes universitarios. Ciencia e Interculturalidad, 17(2), 38-45. DOI: http:// dx.doi.org/10.5377/rci.v19i2.3119

Flores, W., O., \& López, E. (2016). Recursos didácticos y tecnológicos para la enseñanza de la integral definida en el modelo de Universidad Comunitaria Intercultural. Ciencia e Interculturalidad, 18(1), 63-78. DOI: http://dx.doi.org/10.5377/rci. v18i1.3050

Godino, J. (2013). Indicadores de la idoneidad didáctica de procesos de enseñanza y aprendizaje de las matemáticas. Cuadernos de Investigación y Formación en Educación Matemática, 11, 111-132.

Hernández, R., Fernández, C., \& Baptista, M. (2010). Metodología de la investigación. México: McGra-Hill.

Jarquin, H. (2009). Programa de estudio de matemáticas de educación secundaria de 7, 8 y 9 grado. Managua: MINED. 
URACCAN. (2007). Currículo de la Licenciatura en Ciencias de la Educación con Mención en Matemáticas. RACCS: URACCAN.

Villa, A., Campo, L., Arranz, S., Villa, O., \& García, A. (2013). Valoración del profesorado de magisterio sobre el aprendizaje basado en competencias implantada. Profesorado. Revista Currículum y Formación del Profesorado, 17(3), 35-55.

Zabalza, M. (2001). Evaluación del aprendizaje en la universidad. En A. García Varcárcel, Didáctica Universitaria . Madrid: La Muralla. 